\title{
Parameter estimation of coalescing supermassive black hole binaries with LISA
}

\author{
K.G. $\operatorname{Arun}^{1, *}$ \\ ${ }^{1}$ Raman Research Institute, Bangalore 560 080, India
}

(Dated: October 30, 2018)

\begin{abstract}
Laser Interferometer Space Antenna (LISA) will routinely observe coalescences of supermassive black hole $(\mathrm{BH})$ binaries up to very high redshifts. LISA can measure mass parameters of such coalescences to a relative accuracy of $10^{-4}-10^{-6}$, for sources at a distance of $3 \mathrm{Gpc}$. The problem of parameter estimation of massive nonspinning binary black holes using post-Newtonian (PN) phasing formula is studied in the context of LISA. Specifically, the performance of the 3.5PN templates is contrasted against its $2 \mathrm{PN}$ counterpart using a waveform which is averaged over the LISA pattern functions. The improvement due to the higher order corrections to the phasing formula is examined by calculating the errors in the estimation of mass parameters at each order. The estimation of the mass parameters $\mathcal{M}$ and $\eta$ are significantly enhanced by using the 3.5PN waveform instead of the $2 \mathrm{PN}$ one. For an equal mass binary of $2 \times 10^{6} M_{\odot}$ at a luminosity distance of $3 \mathrm{Gpc}$, the improvement in chirp mass is $\sim 11 \%$ and that of $\eta$ is $\sim 39 \%$. Estimation of coalescence time $t_{c}$ worsens by $43 \%$. The improvement is larger for the unequal mass binary mergers. These results are compared to the ones obtained using a non-pattern averaged waveform. The errors depend very much on the location and orientation of the source and general conclusions cannot be drawn without performing Monte Carlo simulations. Finally the effect of the choice of the lower frequency cut-off for LISA on the parameter estimation is studied.
\end{abstract}

PACS numbers: 04.30.Db, 04.25.Nx, 04.80.Nn, 95.55.Ym

\section{INTRODUCTION}

Most of our near-by galaxies harbour supermassive black holes $(\mathrm{BH})$ at their centre [1]. If this is the case, then merger of such galaxies would produce a binary system composed of two supermassive BHs. Simulations indicate that there could be primordial supermassive BH binaries at the centres of the first galaxies 2]. An understanding of the formation and evolution of these binaries is very important from the view point of cosmology and structure formation. Many of these binaries coalesce under gravitational wave $(\mathrm{GW})$ radiation reaction within Hubble time. Supermassive $\mathrm{BH}$ binaries in the mass range $10^{4}-10^{7} M_{\odot}$ will emit gravitational waves $(\mathrm{GW})$ of frequency $10^{-4}-10^{-1} \mathrm{~Hz}$ during its adiabatic inspiral phase which can be observed by the proposed space-borne GW missions such as LISA [3, 4] with high $(\sim$ a few thousands) signal to noise ratio (SNR) up to very high redshifts $(\sim 10)$.

Different authors have investigated the implications of these observations in the context of astrophysics, cosmology and testing general relativity and its alternatives. LISA observations of $\mathrm{BH}$ coalescences can be used to study the growth of BHs as the universe evolved and for mapping the distribution of BHs as function of the redshift [5, [6, 7]. LISA will be able to measure luminosity distances to the sources with an accuracy $\sim 1-10 \%$. If the redshift associated with the event is known by electromagnetic observations, these sources can be used as very high precision standard candles and to study the distance-redshift relation 8, 9]. Ref 10 discussed the potential of LISA to observe binaries containing a $\mathrm{BH}$ in the intermediate mass regime $\left(\sim 10^{3} M_{\odot}\right)$ and use it as a probe of strong field aspects of gravity.

LISA could probe many strong gravitational field effects which are not possible to explore with other observational means. Both inspiral and ring-down GW signals can be used for this. Refs [11, 12] studied the possibility of using the quasi-normal mode oscillations to test the no-hair theorem of general relativity since these modes will be characterized only by the mass and angular momentum of the BH (in general relativity). Hughes and Menou examined another possibility [13. if LISA detects both inspiral and ring-down signals from the same source. By measuring the total mass independently from both the signals one can estimate the mass difference which will be the mass-energy lost due to GWs. They suggested that an extension of this idea including spin effects could in principle test the BH area theorem. Further, inspiral of a stellar mass BH into a SMBH will be another interesting source for LISA using which many properties of the central SMBH can be probed including the possibility to map the spacetime following the geodesics of the stellar mass BH (See e.g. 14, 15]) and measuring the multipole moments of the spacetime.

LISA will provide an unique opportunity to test general relativity and its alternatives. Will and his co-workers have

*Electronic address: arun@rri.res.in 
discussed the potential of LISA to test general relativity as well as its alternatives like Brans-Dicke theory and massive graviton theories [16, 17]. Recently this issue was discussed in a more realistic scenario of spinning binaries using 2PN phasing [6, 7]. Blanchet and Sathyaprakash proposed another test based on post-Newtonian (PN) GW phasing formula by measuring the 1.5PN GW tail effect and showing how it can be used as a test of general relativity [18, 19]. This proposal was recently generalized to higher order terms in the phasing formula by Arun et al [20] and it was argued that such a test would allow one to probe the nonlinear structure of gravity [21].

A very accurate parameter extraction scheme is central to performing all these analyses. A parameter estimation scheme based on matched filtering, similar to that for the ground based detectors such as LIGO and VIRGO, will be employed for LISA also. An efficient matched filtering would in turn demand a very accurate model of the gravitational waveform. In order to compute the gravitational waveform from a compact binary system, one solves the two-body problem in general relativity perturbatively using different approximation schemes since no exact solutions for this problem exist till date. The final waveform can be expressed as a post-Newtonian expansion which is a power series in $v / c$ where $v$ is the gauge independent velocity parameter characterising the source (See Ref. [22] for an exhaustive review on the formalism). In our notation $\frac{v}{c}$ refers to half a PN order.

Since the information about the phase is more important for the process of matched filtering, one uses a simplified model of the inspiral waveform (the so called restricted waveform) where phase is modelled to a high PN order retaining the Newtonian amplitude. In doing so, one is neglecting the effect of other harmonics [23, 24] in the amplitude of the wave and also the higher order PN corrections to the dominant harmonic at twice the orbital frequency. In the present study, we deal only with the restricted waveform in the Fourier domain obtained using stationary phase approximation. The phasing formula for nonspinning binaries, is presently complete up to 3.5PN order [25, 26, 27, 28].

The implications of the higher PN order phasing in the context of parameter estimation problem has been investigated by different authors. Based on the framework set up by Refs [29, 30], Cutler and Flanagan [31] investigated the importance of the 1.5PN phasing formula [32]. The effect of including spin-orbit coupling parameter at 1.5PN order into the space of parameters was one of interesting issues addressed. Two independent works by Królak et al. 33. and Poisson and Will [34] analysed the problem of parameter estimation using the 2PN phasing formula of Ref. [25]. Inclusion of the spin-spin coupling term at $2 \mathrm{PN}$ and its effect on the errors of other parameters was the focus of their analysis.

Recently Arun et al. 35] investigated the effect of the 2.5, 3 and 3.5PN terms for the parameter estimation of nonspinning binaries (a similar work was carried out independently by Berti and Buonanno [36]). Using covariance matrix calculations, they inferred that by employing the $3.5 \mathrm{PN}$ phasing instead of the $2 \mathrm{PN}$ one, the improvement in estimation of errors in chirp mass and symmetric mass ratio can be as high as $19 \%$ and $52 \%$ respectively for the ground based detectors such as LIGO and VIRGO.

Cutler was one of the first to address the problem of parameter estimation in the LISA context 37]. He used the 1.5PN waveform including the spin-orbit effect and studied the estimation of errors associated with the mass parameters as well as distance and angular resolution of the binary. Seto investigated the effect of finite arm length of LISA using 1.5PN phasing [38]. Vecchio revisited the parameter estimation with the 1.5PN waveform [39] where he used the waveform for circular orbit but with "simple precession" (as opposed to the non-precessing case of [37]) and examined the implications of it for the estimation of distance and angular resolution. Various aspects of the 2PN parameter estimation, such as the spin-spin coupling, was investigated by different authors [5, 6, 7]. Ref [6] studied the effect of spin terms in testing alternate theories of gravity with the LISA observations. Refs [5, 7] also addressed the issue of mapping the merger history of massive BHs using LISA observations in the 2PN context. While all these calculations are within the restricted waveform approximation where the PN corrections to the amplitude is completely neglected, there have been investigations about the effect of including these amplitude corrections in the context of parameter estimation 40, 41, 42, 43.

Other than the covariance matrix approach, which is valid only in the high SNR limit, there have been proposals in literature addressing the parameter estimation problem using Monte-Carlo methods. In Ref [44], the authors compared the error estimates obtained using the covariance matrix with the Monte-Carlo simulations. Recently parameter estimation schemes based on Bayesian statistics using Markov chain Monte-Carlo (MCMC) method also has been proposed and implemented in the ground based detector context [45, 46] and the LISA case [47, 48]. Using 2PN waveform Ref [4] found that posterior parameter estimation distribution of the extrinsic parameters obtained using MCMC methods are in excellent agreement with those computed using Fisher matrix whereas there is a systematic overestimate by Fisher matrix for the intrinsic parameters.

In the present work we extend using the covariance matrix formalism, the parameter estimation problem for supermassive BH binary inspirals in LISA and study the implications of higher PN order terms. Coalescences of BHs of masses $10^{4}-10^{7} M_{\odot}$ at a luminosity distance of $3 \mathrm{Gpc}$ are considered. We assume LISA will observe these events for one year duration. Using the 3.5PN phasing we calculate the errors associated with the estimation of the mass parameters and coalescence time and compare them with the corresponding $2 \mathrm{PN}$ results. We also study, for chosen source locations and orientations, the effect of orbital motion for parameter estimation by comparing these 
results with the other two cases: one where LISA is assumed to be a single interferometer and another when LISA is considered to be a two detector network. Conclusions drawn from the exercise above is far from general and have to be supplemented in future by rigorous Monte Carlo simulations.

The rest of the paper is organized as follows. Sec. II discusses all the necessary inputs required for the paper such as a brief introduction to parameter estimation using covariance matrix, noise model for LISA, model for the waveform and some other conventions followed in the paper. Secs III and IV discuss the main results and their implications and Sec. $\mathrm{V}$ provides the summary and future directions.

\section{PARAMETER ESTIMATION FOR LISA WITH 3.5PN PHASING}

\section{A. Parameter estimation using the covariance matrix}

We summarize the theory of parameter estimation in the context of Gaussian random detector noise, addressed in the GW context first by Finn and Chernoff [29, 30] and implemented in detail by Cutler and Flanagan [31]. Let us assume an inspiral GW signal is detected meeting the necessary detection criteria and one needs to extract the intrinsic and extrinsic parameters from the signal by matched filtering.

For sources like inspiralling compact binaries, where a prior source modelling is possible to predict the gravitational waveform, matched filtering is an ideal method both for detection as well as parameter estimation of the signal [49]. In matched filtering, the detector output is filtered using a bank of theoretical templates with different signal parameters. The parameters of the template which obtains the best signal-to-noise ratio (SNR) gives the "measured" values of the signal parameters. These values, in general, will be different from the "actual" values due to the presence of noise. The problem of parameter estimation addresses the question of how close are the measured values to the actual ones and what the associated errors are in the estimation of different parameters. For a given signal, different realizations of noise lead to different sets of best-fit parameters of the signal. When the background noise is a stationary, random, Gaussian process, at high enough SNRs the best-fit values of the parameters have a Gaussian distribution centered around the actual values of the parameters.

If $\lambda^{i}$ denotes the actual value of the parameters and $\lambda^{i}+\Delta \lambda^{i}$, the measured value, then the root mean square difference $\Delta \lambda^{i}$ obeys a Gaussian distribution: $p\left(\Delta \lambda^{i}\right) \propto \exp \left(-\Gamma_{i j} \Delta \lambda^{i} \Delta \lambda^{j} / 2\right)$ where $\Gamma_{i j}$, the Fisher information matrix constructed from the Fourier domain representation of the waveform, is given by

$$
\Gamma_{i j}=2 \int_{f_{\text {in }}}^{f_{\text {fin }}} \frac{\tilde{h}_{i}^{*}(f) \tilde{h}_{j}(f)+\tilde{h}_{i}(f) \tilde{h}_{j}^{*}(f)}{S_{h}(f)} d f .
$$

Here, $\tilde{h}_{i}(f):=\partial \tilde{h}(f) / \partial \lambda^{i}, \tilde{h}(f)$ is the Fourier domain gravitational waveform and $S_{h}(f)$ is the (one-sided) noise power spectral density of the detector. $f_{\text {in }}$ and $f_{\text {fin }}$ denote the lower and upper limits of integration which is defined later in the section. It also follows that the root-mean-square errors are given by $\sigma^{i}=\sqrt{\Sigma^{i i}}$, where $\Sigma=\Gamma^{-1}$ is called the covariance matrix. The non-diagonal elements of the covariance matrix are related to the correlation coefficient between two parameters $\lambda^{i}$ and $\lambda^{j}: c^{i j}:=\frac{\Sigma^{i j}}{\sqrt{\Sigma^{i i} \Sigma^{j j}}}$. Repeated indices are not summed over in the above expressions. Finally the SNR can be expressed in terms of the Fourier domain signal $\tilde{h}(f)$ as

$$
\rho^{2}=4 \int_{f_{\text {in }}}^{f_{\text {fin }}} \frac{|\tilde{h}(f)|^{2}}{S_{h}(f)} d f .
$$

In the present case the $\lambda^{i}$ denoting our chosen set of parameters are given by $\left\{t_{c}, \phi_{c}, \mathcal{M}, \eta, D_{L}, \bar{\phi}_{S}, \bar{\phi}_{L}, \bar{\theta}_{S}, \bar{\theta}_{L}\right\}$. In the case where pattern functions are not included the above set reduces to $\left\{t_{c}, \phi_{c}, \mathcal{M}, \eta\right\}$. The additional elements of the parameter set denotes the distance, orientations and locations of the source in the sky specified with respect to the fixed solar system based coordinate system.

In the above integrals, the upper limit of integration is $f_{\text {fin }}=\operatorname{Min}\left[f_{\text {lso }}, f_{\text {end }}\right]$, where $f_{\text {lso }}$ is the frequency of the innermost stable circular orbit for the test particle case, $f_{\text {lso }}=\left(6^{3 / 2} \pi m\right)^{-1}$ and $f_{\text {upper }}$ corresponds to the upper cut-off of the LISA noise curve $f_{\text {end }}=1 \mathrm{~Hz}$. We have chosen the lower limit of frequency $f_{\text {in }}=\operatorname{Max}\left[f_{\text {in }}, f_{\text {lower }}\right]$ where $f_{\text {in }}$ is calculated by assuming the signal to last for one year in the LISA sensitivity band and $f_{\text {lower }}$, the low frequency cut-off for LISA noise curve, is assumed to be $10^{-5} \mathrm{~Hz}^{1}$. This is a rather optimistic choice for the lower cut-off for

\footnotetext{
1 Another way of choosing the limits of integration is to calculate the time over which the signal will last once it enters the LISA band. See [5] for example, where the duration of the signal is calculated using the expression for $t(f)$. It assumes $f_{\text {lower }}=10^{-4} \mathrm{~Hz}$ and 3 year mission time for LISA.
} 
LISA and in Sec. IIIG we estimate its effect on parameter estimation by comparing it to the more modest choice of $10^{-4} \mathrm{~Hz}$.

We follow the noise model of LISA as given in Sec. IIC of Ref [6] which is a slightly modified version of [50]. The noise spectral density consists of a non-sky averaged part [50] and confusion noise due to the galactic and extra galactic white dwarf binaries [50, 51, 52]. The explicit expressions for these components can be found in Eqs (2.28)-(2.32) of Ref [6].

\section{B. LISA detector configuration and the waveform model}

LISA is a three-arm interferometer where each arm has a length of $5 \times 10^{6} \mathrm{~km}$. These arms form an equilateral triangle and move in a heliocentric orbit with a $20^{\circ}$ lag to the earth and the plane of the detector tilted at $60^{\circ}$ with respect to the ecliptic (See [3, 37] for details). LISA with three arms is essentially equivalent to a pair of two-arm detectors capable of simultaneously measuring the two polarizations of the incoming gravitational wave. We consider two cases: one where we assume the estimation of mass parameters is not affected because of their correlations with the angular variables and second when we estimate the associated errors with angular variables and luminosity distance of the source. Since the information about the angular variables are encoded in the so-called pattern functions which describes the orbital motion of LISA, in the first case we use a waveform which is averaged over the pattern functions. In the second case, we do not average over the pattern functions and use the information from the LISA orbital motion to discuss the estimation of angular resolution and luminosity distance to the source. Further, in the second case we consider cases when (i) LISA is a single two arm-detector and (ii) as a two detector network in order to understand the effect of network configuration for parameter estimation. The LISA antenna patterns, describing its orbital motion, is given in [37] which is used for the present study (also see Appendix A of Ref [6] for these expressions).

Unlike the ground based detectors where the two arms have an angle $90^{\circ}$, the LISA arms are at $60^{\circ}$. As shown by Cutler [37], the relative strain amplitude (which is the gravitational waveform) in the LISA case can be simply be related to the $90^{\circ}$ interferometer case by multiplying the latter by a factor $\sqrt{3} / 2$. Using this input, the Fourier domain waveform within the stationary phase approximation can be written down as [6, 37]

$$
\begin{aligned}
\tilde{h}_{\alpha}(f) & =\frac{\sqrt{3}}{2} \mathcal{A} f^{-7 / 6} e^{\mathrm{i} \psi(\mathrm{f})}, \quad \alpha=\mathrm{I}, \mathrm{II}, \\
\mathcal{A} & =\frac{1}{\sqrt{30} \pi^{2 / 3}} \frac{\mathcal{M}^{5 / 6}}{D_{\mathrm{L}}},
\end{aligned}
$$

where $\alpha$ labels the interferometer, $f$ the GW frequency and $\mathcal{M}$, the chirp mass which is related to total mass $m=m_{1}+m_{2}$ and symmetric mass ratio $\eta=m_{1} m_{2} / m^{2}$ by $\mathcal{M}=\eta^{3 / 5} m$. The luminosity distance to the source is denoted by $D_{L}$. The GW phase $\psi(f)$ appearing in the formula is completed up to 3.5PN [27, 28, 53] and its Fourier domain representation is given in $[54,55$. . We find it more convenient to write it as

$$
\psi(f)=2 \pi f t_{c}-\phi_{c}+\sum_{k=0}^{k=7} \alpha_{k} v^{k}
$$

where $v=(\pi m f)^{1 / 3}$ is the PN variable which is related to gauge independent source velocity in system of units where $G=1=c$ which we follow henceforth in the paper. Eq (3.4) of [35] gives the $\alpha_{k}$ for different values of $k=0 \cdots 7$.

In the case where we do not average the pattern functions, the waveform can be written as [6]

$$
\tilde{h}_{\alpha}(f)=\frac{\sqrt{3}}{2} \mathcal{A} f^{-7 / 6} e^{i \Psi(f)}\left\{\frac{5}{4} \tilde{A}_{\alpha}(t(f))\right\} e^{-i\left(\varphi_{p, \alpha}(t(f))+\varphi_{D}(t(f))\right)},
$$

where $\varphi_{p, \alpha}(t(f))$ and $\varphi_{D}\left(t(f)\right.$ are the polarization phase and Doppler phase respectively [37]. $\tilde{A}_{\alpha}(t(f))$ correspond to the amplitude modulations induced by the LISA's orbital motion. $\tilde{A}_{\alpha}(t(f))$ and $\varphi_{p, \alpha}(t(f))$ depends on the pattern functions $F_{+}^{\alpha}(t)$ and $F_{\times}^{\alpha}(t)$ and hence vary with time. For the explicit expressions for $\varphi_{p, \alpha}$ and $\varphi_{D}$, we refer the readers to Refs [6, 37]. For $3.5 \mathrm{PN}$ accurate expression for $t(f)$ we use the following relation

$$
2 \pi t(f)=\frac{d \psi(f)}{d f} .
$$

This can be rewritten as

$$
t(f)=t_{c}-\sum_{k=0}^{7} t_{k}^{v} v^{k}
$$


and values of $t_{k}^{v}$ is given in Refs $[54,55]$ which can readily be used.

For calculations where LISA is assumed to be a two detector network, we calculate the SNR and Fisher matrix using

$$
\begin{aligned}
& \rho^{\text {Network }}=\sqrt{\rho_{\mathrm{I}}^{2}+\rho_{\mathrm{II}}^{2}}, \\
& \Gamma_{a b}^{\text {Network }}=\Gamma_{a b}^{\mathrm{I}}+\Gamma_{a b}^{\mathrm{II}} .
\end{aligned}
$$

The errors for the two detector case are obtained inverting the total Fisher matrix following the procedure outlined in Sec. IIA

Throughout the paper we assume a cosmological model with zero spatial curvature $\Omega_{\kappa}=0, \Omega_{\Lambda}+\Omega_{M}=1$ and Hubble's constant to be $H_{0}=70 \mathrm{~km} \mathrm{~s}^{-1} \mathrm{Mpc}^{-1}$. $\Omega_{\Lambda}$ and $\Omega_{M}$ refers to the contributions to the total density from matter and cosmological constant. The luminosity distance is given by

$$
D_{L}=\frac{1+z}{H_{0}} \int_{0}^{z} \frac{d z^{\prime}}{\left[\Omega_{M}\left(1+z^{\prime}\right)^{3}+\Omega_{\Lambda}\right]^{1 / 2}},
$$

where $z$ denotes the redshift of the source.

We calculate the Fisher matrix for the different configurations of LISA using the corresponding waveforms and invert it to get the covariance matrix. The elements of the covariance matrix are used for discussing the errors and correlation coefficients of different parameters in the next section. While discussing the trends with the PN orders, its useful to keep in mind that when pattern functions are included, there are additional PN expansions coming from $t(f)$ both in the amplitude and phase, apart from the usual phase $\psi(f)$, which can influence the results.

\section{PARAMETER ESTIMATION WITH PATTERN AVERAGED WAVEFORM}

In this section we discuss the parameter estimation in the LISA case using pattern averaged waveform of Eq (2.3). Parameter estimation with the non-pattern averaged waveform (as in Eq 2.5) is more complex since the estimated errors strongly depend on the location and orientation of the source (see the Sec. IV below) which enter the calculation via the pattern functions. The ideal way to deal with the situation will be to perform Monte Carlo simulations for different binaries located and oriented randomly in the sky [5, 6, 7, 39]. A recent Monte Carlo simulation [6], which addressed the parameter estimation problem using the $2 \mathrm{PN}$ waveform including spin effects, compared the results of their simulation with the result they obtained using pattern averaged waveform (see Tables V, VI and VIII of Ref [6] ). They found that the results in both the cases are in excellent agreement. The results presented in this section about the improved parameter estimation with the pattern averaged waveform, may hence give a reasonably good idea about the full problem where the LISA pattern functions are included and it is considered to be a two detector network. We emphasize that the results quoted here have to be supported by Monte Carlo simulations similar to Ref [6] (see concluding remarks in Sec. V].

\section{A. Improved parameter estimation of equal mass binaries with the 3.5PN phasing}

We discuss the performance of the $3.5 \mathrm{PN}$ restricted waveform from the parameter estimation point of view for the pattern-averaged case discussed above. Our aim is to study the variation of errors in different parameters with the total observed mass ${ }^{2}$ of the binary for different PN orders. This would not only give us an idea of the improvement brought in by the use of higher order phasing but also about the convergence of the PN series for the problem of parameter estimation. We have checked our codes by reproducing the results at $2 \mathrm{PN}$ with that in Table III and $\mathrm{V}$ of Ref [6] for the nonspinning case. The important results of our study are discussed in detail in what follows. The errors in estimation of different parameters for a $2 \times 10^{6} M_{\odot}$ binary at $3 \mathrm{Gpc}$ is provided in Table I for different PN orders in the phasing.

\footnotetext{
${ }^{2}$ By total mass, we always refer to the total redshifted mass $m^{\prime}(1+z)$, where $m^{\prime}$ is the actual source mass and $z$ is the redshift of the source. This is the mass parameter that is measured by the GW observations.
} 

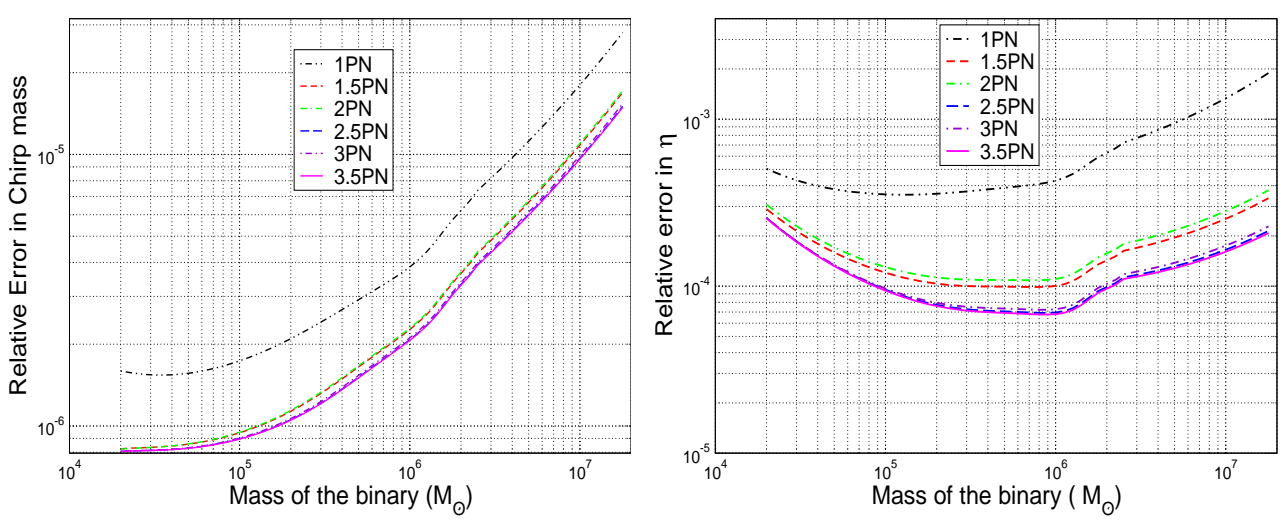

FIG. 1: Variation of errors with the total observed mass for different PN order restricted waveforms for LISA. Pattern averaged waveform is used. The convergence of the results is evident in both the cases. Sources are assumed to be at $3 \mathrm{Gpc}$.

\section{B. Improvement in estimation of mass parameters and $\mathrm{PN}$ convergence}

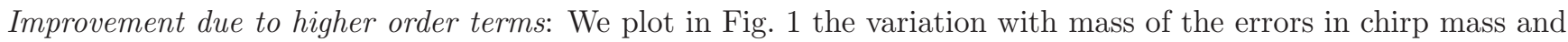
$\eta$ for different PN orders. There is significant improvement in the estimation by the use of the 3.5PN phasing instead of the $2 \mathrm{PN}$ one especially for more massive systems. For a prototypical system of a binary BH each of mass $10^{6} M_{\odot}$, we find that the chirp mass and $\eta$ improve by $11 \%$ and $39 \%$ respectively. Improvement is higher for more massive systems. For a $2 \times 10^{7} M_{\odot}$ binary, the chirp mass and $\eta$ improves by $14 \%$ and $45 \%$. They are similar to the results for the ground based detectors as discussed in [35] but at an entirely different mass range. For a typical binary in its sensitivity band, LISA will be able to measure chirp mass with an incredibly small fractional accuracy of $\sim 10^{-6}$ and $\eta$ by about $\sim 10^{-4}$.

Variation with mass: The estimation of the chirp mass worsens with increase in total mass of the binary whereas the estimation of $\eta$ improves initially and then decreases. These effects can be understood as follows. When the total mass increases there are two competing effects in action: the increase in errors with mass, since signal lasts for smaller duration, and the variation of SNR with mass, which is a characteristic of the noise curve. For chirp mass the errors increase so rapidly that the variation in SNR does not affect the trend and the errors continue to increase monotonically with mass. For $\eta$, there is trade-off between these two competing effects which accounts for the minimum in the curve.

\section{Errors in coalescence time}

Measuring the time of coalescence of a binary system is important to carry out electro-magnetic observation of the event associated with the binary merger. We discuss the trends in estimation of $t_{c}$ below.

Fig. 2 displays the variation of errors in $t_{c}$ with increasing mass of the source and across the PN orders. The errors in $t_{c}$ show trends similar to that of [35], i.e., with increase in PN order the errors oscillate in a sense opposite to the mass parameters and in going from $2 \mathrm{PN}$ to $3.5 \mathrm{PN}$ there is a net degradation in its estimation, which is about $43 \%$ for the $2 \times 10^{6} M_{\odot}$ system considered. This was explained in Ref [35] based on the correlations between $t_{c}, \mathcal{M}$ and $\eta$. It was noticed that both $c_{\mathcal{M} t_{c}}$ and $c_{\eta t_{c}}$ are positive and follow the same trend as the error in $t_{c}$. Increase in these correlations implied a worsened estimation of $t_{c}$.

\section{Post-Newtonian convergence in the parameter estimation context}

Since the PN series is an asymptotic series, the rate of convergence of the results is a very important issue for detection as well as parameter estimation. We use the word 'convergence' to mean that the difference (in errors) between two consecutive PN orders is smaller as we go to higher orders considered. As remarked in Refs. 31, 34], if the parameter estimation scheme is based on a lower order $(2 \mathrm{PN})$ phasing, the systematic errors due to the absence of higher order terms may be more than the statistical errors caused by the noise. Since we study here the implications of 3.5PN phasing, we examine the convergence of the series based on our results for different PN orders.

As Fig. प reveals, though there will be improvement by using the $3.5 \mathrm{PN}$ phasing instead of the $2 \mathrm{PN}$ one, most of 


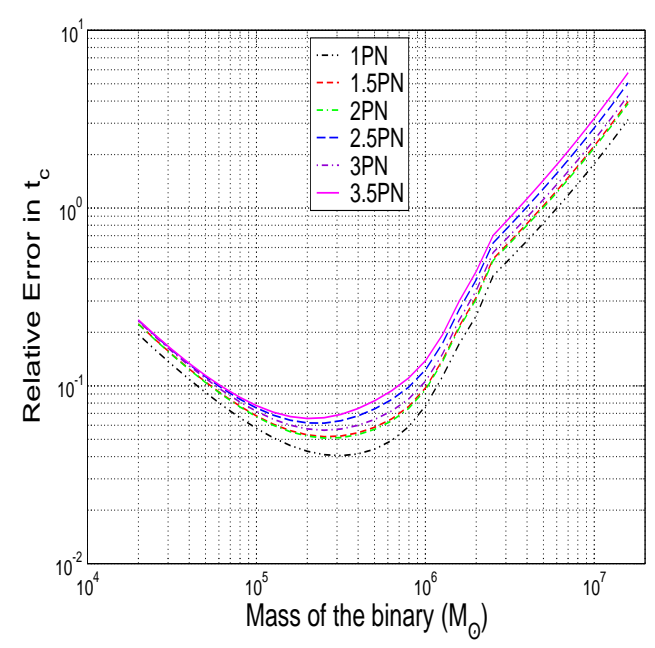

FIG. 2: Variation of errors in $t_{c}$ with the total observed mass for different PN order restricted waveforms for LISA. Pattern averaged waveform is used. The convergence of the results is evident from the plot. Sources are assumed to be at 3 Gpc.

TABLE I: Variation of errors in different parameters and number of GW cycles with PN order. Errors are calculated with the pattern averaged waveform. The system considered is a binary of mass $2 \times 10^{6} M_{\odot}$ at a luminosity distance of 3 Gpc. Most of the features regarding the improvement in parameter estimation, convergence of the PN series and correlation with number of GW cycles are captured in this table.

\begin{tabular}{l|c|c|c|c}
\hline \hline PN Order & $\begin{array}{c}\Delta t_{c} \\
(\mathrm{sec})\end{array}$ & $\begin{array}{c}\Delta \mathcal{M} / \mathcal{M} \\
\left(10^{-6}\right)\end{array}$ & $\begin{array}{c}\Delta \eta / \eta \\
\left(10^{-4}\right)\end{array}$ & $N_{\text {cycles }}$ \\
\hline \hline $1 \mathrm{PN}$ & 0.2474 & 6.217 & 6.287 & 2414.03 \\
$1.5 \mathrm{PN}$ & 0.3149 & 3.648 & 1.427 & 2310.26 \\
$2 \mathrm{PN}$ & 0.3074 & 3.694 & 1.572 & 2305.52 \\
$2.5 \mathrm{PN}$ & 0.3947 & 3.320 & 0.9882 & 2314.48 \\
$3 \mathrm{PN}$ & 0.3435 & 3.377 & 1.033 & 2308.73 \\
$3.5 \mathrm{PN}$ & 0.4399 & 3.300 & 0.9661 & 2308.13 \\
\hline \hline
\end{tabular}

the improvement seems to come from the transition between $2 \mathrm{PN}$ and $2.5 \mathrm{PN}$ after which the series continues to show its characteristic oscillatory behaviour, but with smaller magnitude, suggesting that phasing at orders higher than 3.5PN may not cause much improvement (see Table I).

\section{E. Parameter estimation and Number of GW cycles}

In Ref. 35], the correlation between the improvement in errors across different PN orders and the number of total and useful GW cycles [56] was studied. It was found that though they are good indicators of how the errors at each order vary with the total mass of the system, they alone cannot explain the variation of errors across different PN orders in the context of ground based detectors. We confirm this feature in the LISA context. We recover the results in Table I and II of Ref $[\underline{6}$ as a check of our calculation. Table I shows how the errors and total GW cycles vary with increasing PN orders. One would expect an improved (worsened) estimation if number of GW cycles increases (decreases) between two consecutive PN orders if they were solely responsible for the trends. From the table it is clear that from $1 \mathrm{PN}$ to $1.5 \mathrm{PN}$ and $1.5 \mathrm{PN}$ to $2 \mathrm{PN}$, the errors in chirp mass and $\eta$ do not conform to the above expectation. The same is the case in going from $3 \mathrm{PN}$ to $3.5 \mathrm{PN}$. Finally, trends in $t_{c}$ being opposite to that of the other two mass parameters lead one to conclude once again that the number of GW cycles is not sufficient to understand the variation of errors with PN orders.

Irrespective of whether the total number of GW cycles is very high $\left(\sim 10^{5}\right)$ (as in the case of LISA) or low $(\sim$ hundreds) (as for the ground based detectors) the PN trends in parameter estimation are too complicated to be explained solely in terms of this. However the variation of errors with mass can be understood on the basis of number 
of the cycles and variation in SNR (again, not in terms of only one of them).

\section{F. Parameter estimation for unequal mass binaries}

Lastly we perform a similar analysis for unequal mass systems where $\eta<0.25$. For larger mass ratios $\left(\eta \simeq 10^{-5}\right.$ or smaller in our case), the Fisher matrix becomes ill-conditioned [6] and hence we restrict ourselves to the inspiral of a binary consisting of an intermediate mass $\mathrm{BH}(\mathrm{IMBH})$ and a SMBH rather than stellar mass-SMBH inspirals. An IMBH of $10^{4} M_{\odot}$ inspiralling into a SMBH of $10^{6} M_{\odot}$ constitutes our prototypical system. This system at a distance of $3 \mathrm{Gpc}$ will have a SNR of a few hundreds. We find that the improvement due to the inclusion of higher order terms is more dominant here than for the equal mass binary case. For the prototypical system considered above, we find an improvement of $11 \%$ for the chirp mass and $52 \%$ for $\eta$ in the pattern-averaged case. For a $10^{4}-10^{7} M_{\odot}$ binary, where SNR is $\simeq 100$, the improvement is even more: $20 \%$ and $62 \%$ respectively ${ }^{3}$.

This larger improvement for the unequal mass binaries is not a special feature of the LISA noise curve; for the ground based detectors also a similar feature exists. But unlike in the LISA case, where many such unequal mass binaries are astrophysically plausible, for the ground based detectors such sources are not prototypical.

\section{G. Effect of low frequency cut-off chosen}

All the calculations so far, and hence the results, have been based on the optimistic possibility that the low frequency sensitivity of LISA can be extrapolated from $10^{-4} \mathrm{~Hz}$ to $10^{-5} \mathrm{~Hz}$. As argued in Ref [a] , this significantly improves the parameter estimation. We quantify the effect of this choice of lower cut-off by comparing our previous results with the one where LISA is assumed to be 'blind' below $10^{-4} \mathrm{~Hz}$.

As discussed in Sec. [A we have chosen the lower limit of integration in all the calculations assuming that the system is observed for one year before coalescence, when $f=f_{\text {lso }}$. By this procedure, the lower cut-off for a $2 \times 10^{5.5} M_{\odot}$ binary is about $10^{-4} \mathrm{~Hz}$. This means for systems with masses higher than $2 \times 10^{5.5} M_{\odot}$ (and hence a lower $f_{\text {lso }}$ ) the lower limit of integration for one year observation time will be less than $10^{-4} \mathrm{~Hz}$. If we assume LISA is not sensitive to signals below $10^{-4} \mathrm{~Hz}$, these systems will be observed effectively for less than a year leading to a significant decrease in the number of GW cycles and consequent degradation in parameter estimation (for a $2 \times 10^{6} M_{\odot}$ binary, a lower cut-off of $10^{-5} \mathrm{~Hz}$ will give $2308 \mathrm{GW}$ cycles as opposed to 608 if the cut-off was $10^{-4} \mathrm{~Hz}$ ). Thus for binaries whose masses are higher than $2 \times 10^{5.5} M_{\odot}$, the choice of lower cut-off frequency will affect the results reported here. Fig. 3 displays the variation of $2 \mathrm{PN}$ and $3.5 \mathrm{PN}$ errors in chirp mass and $\eta$ corresponding to the two different lower frequency cut-offs we have chosen. Indeed, as is evident from the plot, the errors start to deviate for binaries whose masses are greater than $2 \times 10^{5.5} M_{\odot}$. For a $2 \times 10^{7} M_{\odot}$ system, using $10^{-5} \mathrm{~Hz}$ as cut-off instead of $10^{-4} \mathrm{~Hz}$ improves the estimation of chirp mass by about 150 times and that of $\eta$ by 40 times. These results confirm the need to push to the extent possible the lower frequency sensitivity of LISA.

Regarding the improvement in parameter estimation in going from $2 \mathrm{PN}$ to $3.5 \mathrm{PN}$, calculation with a cut-off of $10^{-4}$ $\mathrm{Hz}$ shows that for a $2 \times 10^{7} M_{\odot}$ binary the difference in going from $2 \mathrm{PN}$ to $3.5 \mathrm{PN}$ would be $22 \%$ and $60 \%$ (as opposed to $13 \%$ and $45 \%$ with $10^{-5} \mathrm{~Hz}$ ) for chirp mass and $\eta$. The number of GW cycles for a cut-off of $10^{-4} \mathrm{~Hz}$ is just 7 whereas with a $10^{-5} \mathrm{~Hz}$ cut-off it is about 540 . Therefore with a cut-off of $10^{-4} \mathrm{~Hz}$, one is only observing the very late inspiral of the system ${ }^{4}$ whereas with a cut-off of $10^{-5} \mathrm{~Hz}$, the inspiral phase is dominant. The significantly larger variation in parameter estimation in going from $2 \mathrm{PN}$ to $3.5 \mathrm{PN}$ with the $10^{-4} \mathrm{~Hz}$ cut-off could be due to the generally accepted fact that higher order terms in the phasing formula are more important as one approaches the last stable orbit.

\section{PARAMETER ESTIMATION WITHOUT PATTERN AVERAGING}

Having discussed in detail the various aspects of parameter estimation using pattern averaged waveform and factors which affect the process, we now turn our attention to the parameter estimation without pattern averaging. Obviously

\footnotetext{
${ }^{3}$ Using a calculation of the number of GW cycles, Ref 57 has emphasized the need for higher order PN modelling of the IMBH-SMBH binaries. The effects of eccentricity could also play an important role in the dynamics of such binaries.

${ }^{4}$ Inspiral waveforms would be inadequate in this studies. Theoretical approximants, e.g. Effective one body [58, 59] may have to be employed to model this phase of the binary's dynamics.
} 

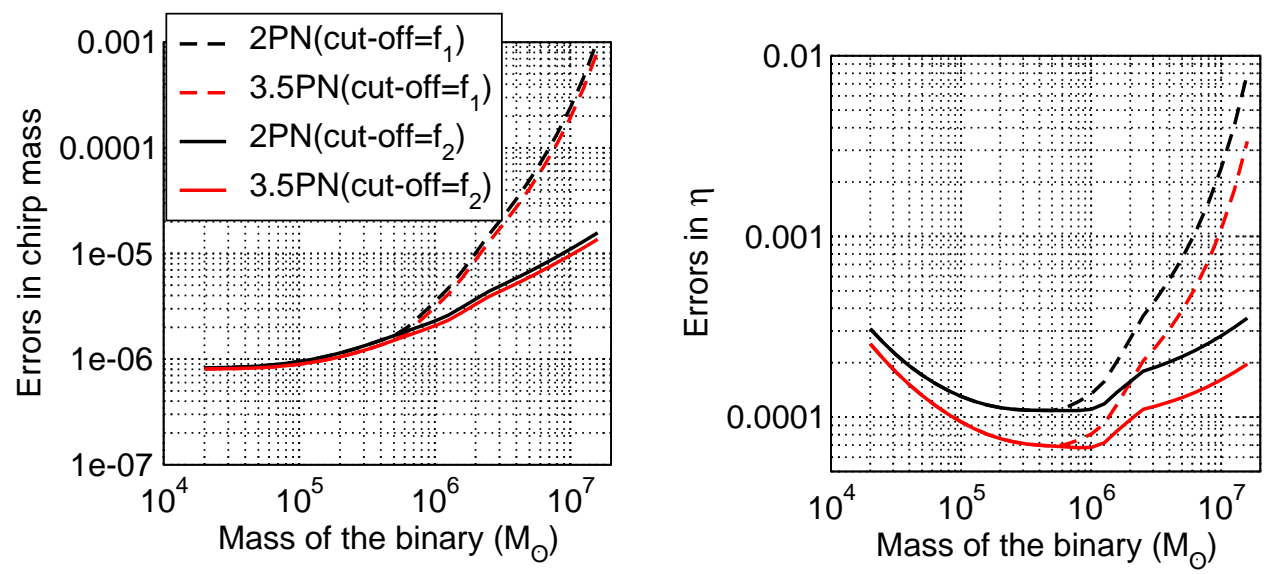

FIG. 3: Variation of errors of chirp mass and $\eta$ with the total observed mass for different PN order restricted waveforms for LISA. Pattern averaged waveform is used. for the two choices of the lower frequency cut-offs $f_{1}=10^{-4} \mathrm{~Hz}_{\mathrm{z} d} f_{2}=10^{-5} \mathrm{~Hz}$. Sources are assumed to be at $3 \mathrm{Gpc}$.

the source's location, orientation and luminosity distance to the source gets added to the space of parameters which was four dimensional in the previous case. Unlike the ground based detectors, LISA can measure the distance, location and orientation of the source with a single detector because of the modulations due to its orbital motion [3, 37]. Besides, using LISA as a two detector network improves the estimation of angular resolution of the source [37]. In this section we will discuss the improvement brought in by the higher order terms using non-pattern averaged waveform for LISA. We check our code, which now includes the pattern functions, by reproducing the results of [37] at 1.5PN with their signal and noise models.

But as mentioned earlier the strong dependence, as we shall discuss, of the errors on the angular variables makes our analysis, which is for selected values of them following Cutler [37], less rigorous. The best way to deal with this situation is to perform Monte Carlo simulations, similar to [5, 6, 6, [39]. However we notice that certain general conclusions can still be drawn from our limited analysis which is the topic of discussion of this section.

\section{A. Comparison of different detector configurations}

We compare the estimation of errors with different detector configurations now. The final errors, in comparison with the pattern averaged case, will depend on (i) the value of SNR corresponding to the set of angles chosen (ii) The change in SNR relative to the pattern averaged case and (iii) worsening of errors due to the introduction of the new parameters.

For the pattern averaged case, since there are no pattern dependent parameters, the total parameter space is essentially 4 dimensional, the parameters being $\left\{t_{c}, \phi_{c}, \mathcal{M}, \eta\right\}$. When we make a transition from the pattern averaged to the non-pattern averaged case, five new parameters $\left\{D_{\mathrm{L}}, \bar{\mu}_{L}, \bar{\mu}_{S}, \bar{\phi}_{L}, \bar{\phi}_{S}\right\}$ corresponding to the distance to the source, its location and orientation are added to the space of parameters. The parameter space is now nine dimensional significantly higher than the earlier four dimensional one. This increase in dimensionality of the parameter space leads to an increase in the errors of the four existing parameters. On the other hand, the introduction of pattern functions results in a change in SNR depending on the four angles chosen. The final picture is a complex interplay of all these features.

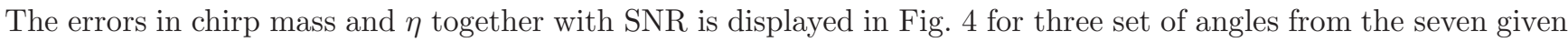
in 37. The three configurations corresponding to the pattern averaged, non-pattern averaged with one detector and the two detector network (without pattern averaging) are considered. As is evident from the plot, the SNR and hence the errors crucially depend on the location and orientation of the source. Also there can be orientations which may have lower SNR than for the pattern averaged case (see third column e.g.). However an interesting point from the figure (and from those runs corresponding to other values of angles which are not displayed) is that among the three effects which influence the parameter estimation without pattern averaging, the value of SNR seems to be the dominant one. Smallest errors in the plot correspond to the configuration with the largest SNR. Between the pattern averaged and the one detector case, the effect of correlations due to the additional parameters play a significant role. For example, in the second column, though one detector case has larger SNR, the errors are smaller for the pattern averaged case for this reason. We do not discuss the percentage improvement arising from the higher order PN terms 

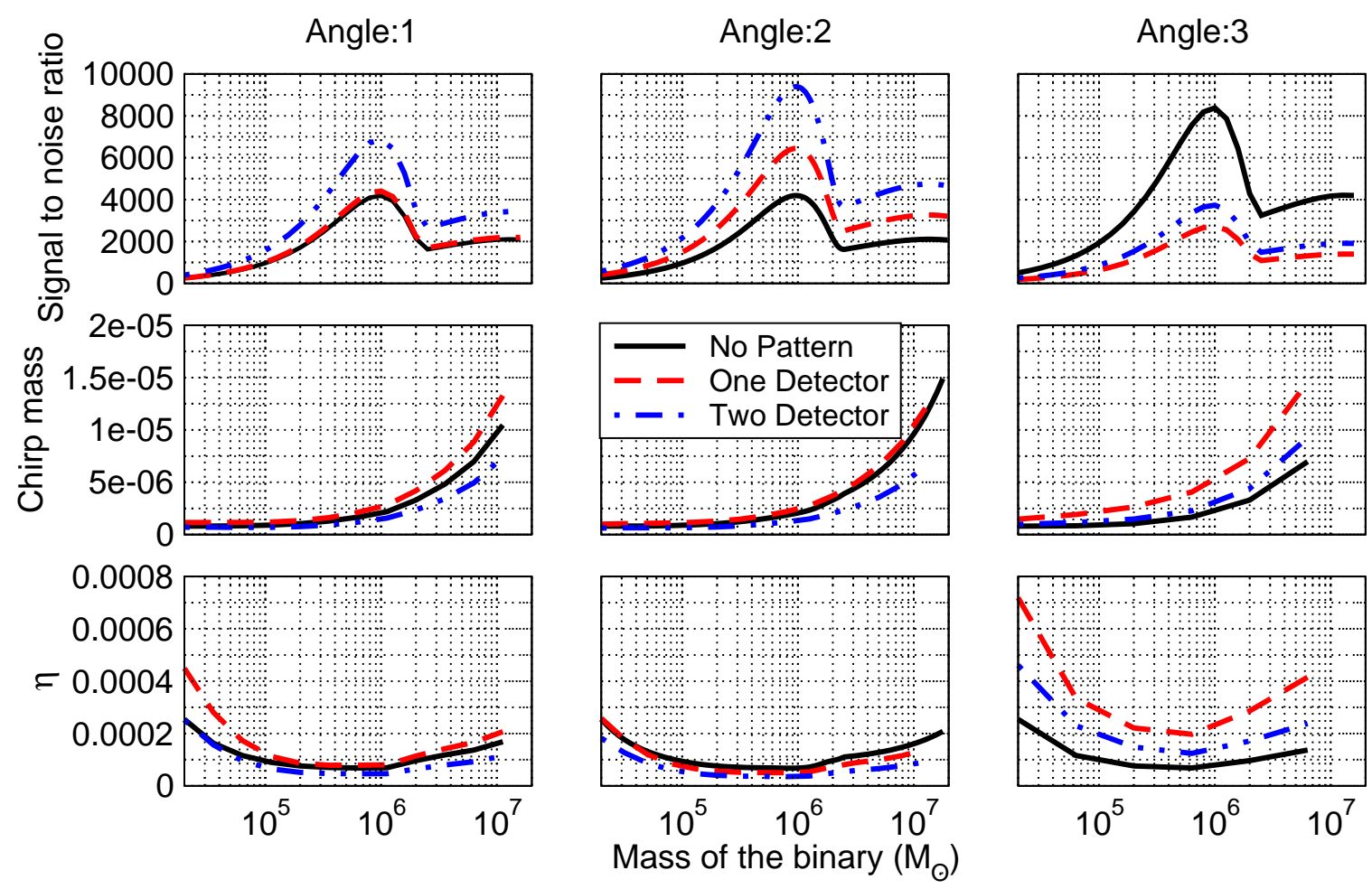

FIG. 4: Variation of the signal to noise ratio, relative errors in chirp mass and relative errors in $\eta$ with the total observed mass of the binary for different choices of location and orientation of the source. Sources are assumed to be at a luminosity distance of $3 \mathrm{Gpc}$ and a non-pattern averaged waveform is used. Angle: 1 corresponds to $\left\{\bar{\mu}_{L}=0.5, \bar{\mu}_{S}=-0.8, \bar{\phi}_{L}=3, \bar{\phi}_{S}=1\right\}$. Angle:2 is $\left\{\bar{\mu}_{L}=0.2, \bar{\mu}_{S}=-0.6, \bar{\phi}_{L}=3, \bar{\phi}_{S}=1\right\}$ and Angle: $3\left\{\bar{\mu}_{L}=0.8, \bar{\mu}_{S}=0.3, \bar{\phi}_{L}=2, \bar{\phi}_{S}=5\right\}$. The errors thus depend very much on the position and orientation of the source in the sky.

since it depends very much on the orientation of the source. Exhaustive Monte Carlo simulations have to be performed to have a detailed understanding of this.

We conclude with a remark about the estimation of angular resolution and distance as observed from the limited set of angles we have considered. The estimation of distance and angular resolution is not improved much because of the additional phasing terms. This is not surprising, since the additional terms in the phasing formula do not carry any information about location or orientation of the binary. One may need to go beyond the restricted waveform model of the waveform in order to achieve this. Some preliminary studies in this regard 40, 41, 42, 43, 60, 61 are consistent with the same. Going beyond the restricted waveform approximation would mean including the amplitude corrections to the waveform from the two GW polarizations, currently completed up to 2.5PN order [23, 24]. This is because the amplitude terms are functions also of the angular positions of the source in the sky, the introduction of which could break different degeneracies, allowing better parameter estimation [40, 41].

\section{SUMMARY AND FUTURE WORKS}

The significance of higher order phasing terms is investigated in the LISA case for different sources using a pattern averaged waveform model. Using the $3.5 \mathrm{PN}$ inspiral waveform instead of the $2 \mathrm{PN}$ one which is currently employed in the GW experiments, mass parameters can be estimated with improved precision for LISA. For an equal mass binary of $2 \times 10^{6} M_{\odot}$ at a luminosity distance of $3 \mathrm{Gpc}$, the improvement in chirp mass is $\sim 11 \%$ and that of $\eta$ is $\sim 39 \%$. The PN series shows convergent behaviour beyond 2.5PN order. The number of GW cycles is a good indicator of how the errors vary with mass but not across different PN orders. The improvement in parameter estimation is more pronounced for binaries with unequal masses. The problem of unequal mass binaries should be revisited with the inclusion of spin effects to higher orders as spin effects play a dominant role in the dynamics of such systems. Inclusion of the spin effects at 2.5PN order in the phase [62, 63] would help us go beyond the results of [6, 39] for the spinning case and will be exciting especially for the equal mass case. The effect of the lower cut-off frequency we have chosen $\left(10^{-5} \mathrm{~Hz}\right)$ on the parameter estimation is studied by comparing the calculation with the more modest cut-off of $10^{-4}$ 
Hz. The estimation of source location and orientation is also studied using the non-pattern averaged waveform for selected source directions and orientations. They do not improve significantly by the use of the restricted 3.5PN template. One may need to go beyond the restricted waveform approximation in order to achieve it. Especially, implications of the full waveform with the $2.5 \mathrm{PN}$ polarizations of Ref [24] together with the 3.5PN phasing of [27] would be an interesting exercise to carry out.

Exhaustive Monte Carlo simulations are required in order to understand the effect of higher PN terms for LISA when the non-pattern averaged waveform is used since the parameter estimation in this case strongly depends on the location and orientation of the source. We plan to take up this problem in the near future.

It may be interesting to employ Markov chain Monte Carlo methods for parameter estimation [47, 48] and compare the results with the ones obtained using the covariance matrix. Extending this to the present context would surely be interesting and should be addressed. Finally, the parameter estimation for the SMBH inspirals may eventually have to be done using the time delay interferometry variables (see 64] and references therein).

The very high accuracy parameter extraction possible with LISA will make it a useful tool of astrophysics and provide thorough probes of strong field aspects of gravity in the future.

\section{Acknowledgments}

The author thanks B R Iyer for useful discussions, valuable comments on the manuscript, and constant encouragement. Conversations with B S Sathyaprakash on issues related the parameter estimation with LISA and his encouragement to pursue this problem are gratefully acknowledged. The author is grateful to Sanjeev Dhurandhar for discussions and comments on the manuscript. The author also thanks M S S Qusailah for useful discussions. I am grateful to the anonymous referee for valuable comments which have improved the presentation significantly.

All the calculations reported in this paper are performed with Mathematica.

[1] D. Richstone, E. A. Ajhar, R. Bender, G. Bower, A. Dressler, S. M. Faber, A. V. Filippenko, K. Gebhardt, R. Green, L. C. Ho, et al., Nature 395, A14 (1998), astro-ph/9810378.

[2] B. Volker and L. Abraham, Astrophys. J 596, 34 (2003), astro-ph/0212400.

[3] P. L. Bender, Lisa: Laser interferometer space antenna for the detection and observation of gravitational waves: Pre-phase: A report (1995), unpublished.

[4] K. Danzmann, Class. Quantum Grav. 14, 1399 (1997).

[5] S. A. Hughes, Mon. Not. R. Astron Soc. 331, 805 (2002), astro-ph/0108483.

[6] E. Berti, A. Buonanno, and C. M. Will, Phys. Rev. D 71, 084025 (2005), gr-qc/0411129.

[7] E. Berti, A. Buonanno, and C. M. Will, Class. Quantum Grav 22, S943 (2005), gr-qc/0504017.

[8] B. F. Schutz, Nature (London) 323, 310 (1986).

[9] D. E. Holz and S. A. Hughes, Astrophys. J 629, 15 (2005), astro-ph/0504616.

[10] M. C. Miller, Astrophys. J 618, 426 (2005), astro-ph/0409331.

[11] O. Dreyer, B. Kelly, B. Krishnan, L. S. Finn, D. Garrison, and R. Lopez-Aleman, Class. Quantum Grav. 21, 787 (2004), gr-qc/0309007.

[12] E. Berti, V. Cardoso, and C. M. Will, Phys. Rev. D 73, 064030 (2006), gr-qc/0512160.

[13] S. A. Hughes and K. Menou, Astrophys. J 623, 689 (2005), astro-ph/0410148.

[14] F. Ryan, Phys. Rev. D 56, 1845 (1997).

[15] N. A. Collins and S. A. Hughes, Phys.Rev. D 69, 124022 (2004), gr-qc/0402063.

[16] C. M. Will, Phys. Rev D 57, 2061 (1998), gr-qc/9709011.

[17] C. M. Will and N. Yunes, Class. Quantum Grav. 21, 4367 (2004), gr-qc/0403100.

[18] L. Blanchet and B. S. Sathyaprakash, Class. Quantum Grav. 11, 2807 (1994).

[19] L. Blanchet and B. S. Sathyaprakash, Phys. Rev. Lett. 74, 1067 (1995).

[20] K. G. Arun, B. R. Iyer, M. S. S. Qusailah, and Sathyaprakash, Class. Quantum Grav. 23, L37 (2006), gr-qc/0604018.

[21] K. G. Arun, B. R. Iyer, M. S. S. Qusailah, and Sathyaprakash (2006), (Phy. Rev. D, To appear), gr-qc/0604067.

[22] L. Blanchet, Living Rev. Rel. 5, 3 (2002), gr-qc/0202016.

[23] L. Blanchet, B. R. Iyer, C. M. Will, and A. G. Wiseman, Class. Quantum Grav. 13, 575 (1996), gr-qc/9602024.

[24] K. G. Arun, L. Blanchet, B. R. Iyer, and M. S. S. Qusailah, Class. Quantum Grav. 21, 3771 (2004), erratum-ibid. 22, 3115 (2005), gr-qc/0404185.

[25] L. Blanchet, T. Damour, B. R. Iyer, C. M. Will, and A. G. Wiseman, Phys. Rev. Lett. 74, 3515 (1995), gr-qc/9501027.

[26] L. Blanchet, Phys. Rev. D 54, 1417 (1996), Erratum Phys. Rev. D71, 129904(E) (2005), gr-qc/9603048.

[27] L. Blanchet, G. Faye, B. R. Iyer, and B. Joguet, Phys. Rev. D 65, 061501(R) (2002), Erratum Phys. Rev. D71, 129902(E) (2005), gr-qc/0105099.

[28] L. Blanchet, T. Damour, G. Esposito-Farèse, and B. R. Iyer, Phys. Rev. Lett. 93, 091101 (2004), gr-qc/0406012. 
[29] L. Finn, Phys. Rev. D 46, 5236 (1992).

[30] L. Finn and D. Chernoff, Phys. Rev. D 47, 2198 (1993).

[31] C. Cutler and E. Flanagan, Phys. Rev. D 49, 2658 (1994).

[32] C. Cutler, T. Apostolatos, L. Bildsten, L. Finn, E. Flanagan, D. Kennefick, D. Markovic, A. Ori, E. Poisson, G. Sussman, et al., Phys. Rev. Lett. 70, 2984 (1993).

[33] A. Królak, K. Kokkotas, and G. Schäfer, Phys. Rev. D 52, 2089 (1995).

[34] E. Poisson and C. Will, Phys. Rev. D 52, 848 (1995).

[35] K. G. Arun, B. R. Iyer, B. S. Sathyaprakash, and P. A. Sundararajan, Phys. Rev. D 71, 084008 (2005), erratum-ibid. D 72, 069903 (2005), gr-qc/0411146.

[36] E. Berti and A. Buonanno (2004), unpublished.

[37] C. Cutler, Phys. Rev. D 57, 7089 (1998).

[38] N. Seto, Phys. Rev. D 66, 122001 (2002), gr-qc/0210028.

[39] A. Vecchio, Phys. Rev. D 70, 042001 (2004).

[40] T. A. Moore and R. W. Hellings, Phys. Rev. D 65, 062001 (2002).

[41] R. W. Hellings and T. A. Moore, Class. Quant. Grav. 20, S181 (2003), gr-qc/0207102.

[42] A. M. Sintes and A. Vecchio, in Rencontres de Moriond:Gravitational waves and experimental gravity, edited by J. Dumarchez (Frontires, Paris, 2000), gr-qc/0005058.

[43] A. M. Sintes and A. Vecchio, in Third Amaldi conference on Gravitational Waves, edited by S. Meshkov (American Institute of Physics Conference Series, 2000), p. 403, gr-qc/0005059.

[44] R. Balasubramanian, B. S. Sathyaprakash, and S. V. Dhurandhar, Phys. Rev. D 53, 3033 (1996), erratum-ibid. D 54, 1860 (1996), gr-qc/9508011.

[45] N. Christensen and R. Meyer, Phy. Rev. D 64, 022001 (2001), gr-qc/0102018.

[46] C. Rover, R. Meyer, and N. Christensen, Bayesian inference on compact binary inspiral gravitational radiation signals in interferometric data (2006), gr-qc/0602067.

[47] N. J. Cornish and E. K. Porter, Mcmc exploration of supermassive black hole binary inspirals (2006), gr-qc/0605085.

[48] E. D. L. Wickham, A. Stroeer, and A. Vecchio, A markov chain monte carlo approach to the study of massive black hole binary systems with lisa (2006), gr-qc/0605071.

[49] L. A. Wainstein and V. D. Zubakov, Extraction of Signals from Noise (Prentice-Hall, Englewood Cliffs, 1962).

[50] L. Barack and C. Cutler, Phy. Rev. D 69, 082005 (2004), gr-qc/0310125.

[51] G. Nelemans, L. R. Yungelson, and S. F. Portegies Zwart, Astron. Astrophysics 375, 890 (2001), astro-ph/0105221.

[52] A. J. Farmer and E. S. Phinney, Mon. Not. Roy. Astron. Soc. 346, 1197 (2003), astro-ph/0304393.

[53] L. Blanchet, B. R. Iyer, and B. Joguet, Phys. Rev. D 65, 064005 (2002), Erratum Phys. Rev. D71, 129903(E) (2005), gr-qc/0105098.

[54] T. Damour, B. R. Iyer, and B. S. Sathyaprakash, Phys. Rev. D 63, 044023 (2001), erratum-ibid. D 72 (2005) 029902, gr-qc/0010009.

[55] T. Damour, B. R. Iyer, and B. S. Sathyaprakash, Phys. Rev. D 66, 027502 (2002), ibid D 66, 027502 (2002), gr-qc/0207021.

[56] T. Damour, B. R. Iyer, and B. S. Sathyaprakash, Phys. Rev. D 62, 084036 (2000), gr-qc/0001023.

[57] E. Berti, Lisa observations of massive black hole mergers: event rates and issues in waveform modelling (2006), astro$\mathrm{ph} / 0602470$.

[58] A. Buonanno and T. Damour, Phys. Rev. D 59, 084006 (1999), gr-qc/9811091.

[59] A. Buonanno and T. Damour, Phys. Rev. D 62, 064015 (2000), gr-qc/0001013.

[60] C. Van Den Broeck, Class. Quantum Grav. 23, L51 (2006), gr-qc/0604032.

[61] C. Van Den Broeck and A. Sengupta (2006), gr-qc/0607092.

[62] G. Faye, L. Blanchet, and A. Buonanno, Higher-order spin effects in the dynamics of compact binaries $i$. equations of motion (2006), article submitted, gr-qc/0605139.

[63] L. Blanchet, A. Buonanno, and G. Faye, Higher-order spin effects in the dynamics of compact binaries ii. radiation field (2006), gr-qc/0605140.

[64] M. Tinto and S. Dhurandhar, Living Rev. Relativity 8, 4 (2005). 